\title{
Sub-Micron Patterning of Polymer Brushes: An Unexpected Discovery from Inkjet Printing of Polyelectrolyte Macroinitiators
}

Adam V. S. Parry, ${ }^{\dagger *}$ Alexander J. Straub, ${ }^{\dagger *}$ Eva M. Villar-Alvarez, ${ }^{\dagger||}$ Takdanai Phuengphol, ${ }^{\dagger}$ Jonathan E. R. Nicoll, ${ }^{\dagger}$ Xavier Lim W. K.,${ }^{\dagger}$ Lianne M. Jordan, ${ }^{\dagger}$ Katie L. Moore ${ }^{\dagger}$ Pablo Taboada, ${ }^{\|}$Stephen G. Yeates ${ }^{\dagger}$ and Steve Edmondson ${ }^{\ddagger+}$

Supporting information

\section{Table of contents}

EXPERIMENTAL

Materials and Methods $\quad$ S2

$\begin{array}{lc}\text { Synthesis of Polymer Macroinitiators } & \text { S3 }\end{array}$

SIJ Jetting of Aqueous Inks and MI Deposition $\quad$ S3

Polymer Brush Growth by SI-ARGET-ATRP $\quad$ S5

SUPPORTING DATA

$\begin{array}{ll}\text { Printing of Pure Macroinitiator Inks } & \text { S7 }\end{array}$

$\begin{array}{lr}\text { Humidity Sensor based on Parallel Printed MI Lines } & \text { S8 }\end{array}$

$\begin{array}{lr}\text { Supporting Data for Sub-Micron Patterning } & \text { S9 }\end{array}$

$\begin{array}{ll}\text { Polymer Brush Patterning } & \text { S17 }\end{array}$

Synthesis of Different Polymers and Block-co-Polymers $\quad$ S20

$\begin{array}{lr}\text { Supporting Information References } & \text { S22 }\end{array}$ 


\section{Experimental}

\section{Materials and Methods}

$\mathrm{N}$-isopropylacrylamide (Sigma Aldrich) was purified by recrystallization from a benzene/hexane solution. 2hydroxyethyl methacrylate (HEMA, 97\%) , copper (II) bromide $\left(\mathrm{CuBr}_{2}, 99.999 \%\right), 2,2$ '-bipyridine (bpy, $\geq 99 \%$ ) and L-ascorbic acid (AA, $\geq 99.0 \%$ ) were purchased from Sigma Aldrich and used as received. All other chemicals and solvents were purchased from Sigma Aldrich or Fisher Scientific and used as received (unless otherwise stated).

Polished, arsenic doped silicon $(n++)$ wafers were purchased from IDB Technologies and used as the substrates in all experiments. Cleaning of silicon substrates was performed by first rinsing and sonicating in acetone, methanol and propan-2-ol then drying in a stream of nitrogen. This was followed by cleaning using an RCA SC-1 procedure, which involved the submersion of the substrates in a mixture of 5 parts water, 1 part hydrogen peroxide solution (Fisher Scientific $30 \mathrm{wt} \%$ ) and 1 part ammonia solution (35 wt\%) at $70{ }^{\circ} \mathrm{C}$ for 15 minutes followed by a water rinse and drying in a stream of nitrogen. Before printing the substrates were exposed to UV-Ozone in a BioForce UV-Ozone ProCleaner Plus for approximately 15 minutes.

Printing was performed on a Super Inkjet SIJ-S050 electrohydrodynamic printer from SIJ Technology. Briefly, this was achieved using the supplied super fine nozzle at a working distance of approximately $20 \mu \mathrm{m}$. Droplet ejection required a minimum of (-ve)300 V, with respect to the grounded substrate and drop-ondemand patterning was achieved by using the pulsed, sinusoidal-type waveform with an amplitude of $50 \mathrm{~V}$.

Surface tension and contact angle measurements were performed on a Kruss DSA-100.

Ellipsometry was conducted using a J A Woollam M2000U spectroscopic ellipsometer. Ellipsometric constants $\Delta$ and $\Psi$ were acquired at three angles of incidence $\left(65^{\circ}, 70^{\circ}\right.$ and $\left.75^{\circ}\right)$ and over wavelengths from $250 \mathrm{~nm}$ to $1000 \mathrm{~nm}$. Data were fitted using the supplied CompleteEASE software. The model used softwaresupplied optical constants for the silicon substrate and a $2 \mathrm{~nm}$ thick $\mathrm{SiO}_{2}$ layer. Initiator and polymer layers were modelled using a Cauchy refractive index approximation and variable thickness. Cauchy $A, B$ and $C$ constants were fitted using the thickest available sample ( $140 \mathrm{~nm}$ polymer) and found to be $A=1.518$, $B=0.00617 \mu \mathrm{m}^{2}, C=1.7015 \times 10^{-5} \mu \mathrm{m}^{4}$ and fixed for all subsequent measurements.

ATR-FTIR spectra were recorded using a Bruker Vertex 70 equipped with a DTGS detector and Harrick Seagull ATR accessory. 64 Spectra were recorded with a resolution of $1 \mathrm{~cm}^{-1}$.

Line profiles were recorded on a Veeco(Bruker) Dektak 8 Stylus profiler with a stylus force of $3 \mathrm{mg}$. Lines approximately $0.5 \mathrm{~mm}$ were measured. The standard diamond stylus with a tip radius of $12.5 \mu \mathrm{m}$ and $45^{\circ}$ cone angle were used. This will lead to significant feature broadening in profilometry line profiles. All quoted feature widths are extracted from AFM only.

Optical microscopy images recorded using a Leica DM2500 M in bright field mode. Images are subjected to linear adjustments of brightness and contrast, for clarity.

Atomic force microscopy was performed on a Park XE-100 in non-contact mode. Images were taken with a $512 \times 512$ pixel resolution at a scan rate of $0.5 \mathrm{~Hz}$. Cantilevers were Bruker RTESP-300 with a nominal spring constant of approximately $40 \mathrm{~N} . \mathrm{m}^{-1}$. Tips were pyramidal with a maximum half-cone angle of $25^{\circ}$. Assuming a typical post-polymerisation feature height of $100 \mathrm{~nm}$, the maximum broadening of sharp features with rectangular cross-section due to tip aspect ratio can be calculated as $93 \mathrm{~nm}$. All AFM images have a left-toright scan direction unless otherwise indicated.

Electron microscopy images were acquired on a Phillips Sirion SEM with beam energy of $5 \mathrm{kV}$ and at a spot size of 3.

High resolution secondary ion mass spectrometry measurements were undertaken with a Cameca NanoSIMS 50L (Gennevilliers, France). The NanoSIMS is capable of imaging the spatial distribution of elements with a resolution down to $50 \mathrm{~nm}$ and at high elemental sensitivity (ppm level). A focused $16 \mathrm{keV} \mathrm{Cs}^{+}$primary ion beam with a beam current of $3.3 \mathrm{pA}(\mathrm{D} 1=3)$ was rastered across the sample to sputter secondary ions from the sample. As these samples were so thin no presputtering was performed to increase the signal intensity. Two sequential images were acquired from the sample, each with a dwell time of $500 \mu \mathrm{s} / \mathrm{px}$ at a pixel resolution of $512 \times 512$ giving a total dose of $6 \times 10^{14} \mathrm{Cs}$ atoms $\mathrm{cm}^{-2}$ across a $30 \times 30 \mu \mathrm{m}$ area. Secondary ions are separated according to their mass-to-charge ratio using a double focusing magnetic mass spectrometer. The detectors were positioned to simultaneously detect ${ }^{16} \mathrm{O}^{-},{ }^{12} \mathrm{C}^{14} \mathrm{~N}^{-},{ }^{28} \mathrm{Si}^{-},{ }^{35} \mathrm{Cl}^{-},{ }^{79} \mathrm{Br}^{-}$and ${ }^{81} \mathrm{Br}^{-}$and were precisely aligned on a thick droplet of the sample. The alignment of the $\mathrm{Br}$ ions was checked by confirming the correct isotope ratio was observed $\left({ }^{79} \mathrm{Br}: 50.69 \%,{ }^{81} \mathrm{Br}\right.$ : 49.31\%). The secondary electron signal was also recorded for each image. An ultra-high vacuum of approximately $9.5 \times 10^{-10} \mathrm{mbar}$ in the sample analysis chamber was maintained during the analysis. NanoSIMS images were analyzed using ImageJ with the OpenMIMS plugin (Harvard, Cambridge, 
MA, USA). The two acquired images were aligned for drift and summed together to improve the counts. Line profiles were taken across the track to show the position of $\mathrm{Br}$ relative to the $\mathrm{Cl}$.

\section{Synthesis of Polymer Macroinitiators}

The cationic poly[2-(dimethylamino)ethyl methacrylate-co-glycerol monomethacrylate] (PDMA-PGMA) based water-soluble macroinitiator, the structure of which is shown in Figure S1, has been reported in detail previously ${ }^{1}$ and is similar to other previously-applied cationic macroinitiators. ${ }^{2,3}$ The PDMA ${ }_{65}-$ PGMA $_{29}$ statistical copolymer precursor $\left(M_{n} 14,880, M_{w} / M_{n} 1.27\right)$ was confirmed as containing a molar ratio of 2 : 0.9 DMA : GMA by ${ }^{1} \mathrm{H}$ NMR. The copolymer precursor had been subsequently derivatized to produce the macroinitiator by complete esterification of the dihydroxy-functional glycerol residues with 2-bromoisobutyryl bromide to provide the ATRP initiator sites, and quaternizing $100 \%$ of the tertiary amine groups with methyl iodide making the copolymer water-soluble and cationic to promote adsorption on the negatively charged silica substrates. ${ }^{2,3}$ The resulting macroinitiator contained approximately 1:1 initiator : positively charged sites.

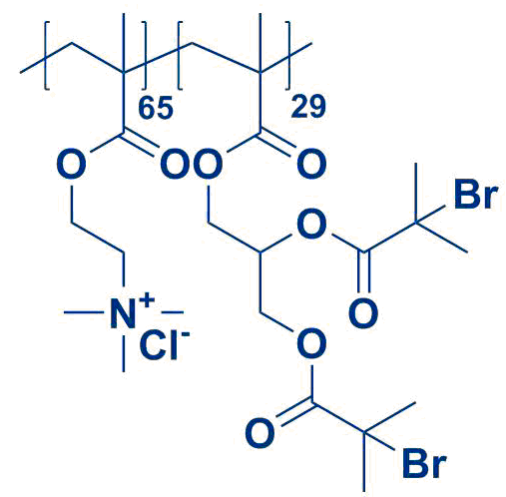

Figure S1. The structure of the cationic PDMA-PGMA based water-soluble macroinitiator.

\section{SIJ Jetting of Aqueous Inks and MI Deposition}

Water alone cannot be printed with the SIJ-printer most likely due to its high surface tension. As such an optimization of MI inks was conducted and solvent/solvent mixtures which lower the surface tension had to be found. Furthermore, fluids which are used in inkjet printing typically have viscosities in the range from $\sim 2$ to $\sim 50 \mathrm{cP}$, because higher viscosities allow for restricted spreading of the ink on the substrate. Moreover, the complete solubility of $\mathrm{MI}$ and MADQUAT must be guaranteed. It should further be mentioned

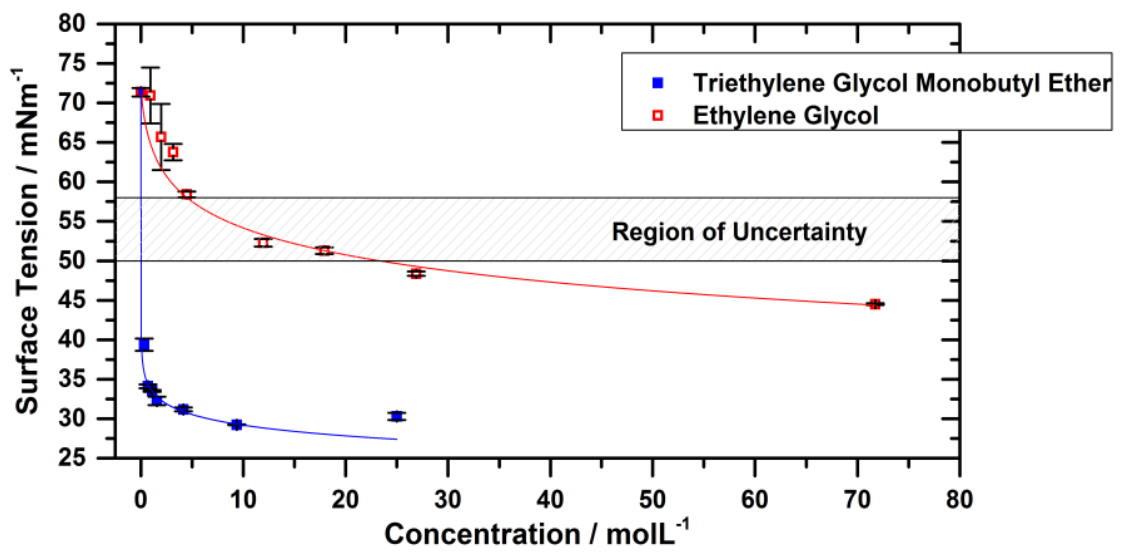

Figure S2. Surface tension variation of water with varying concentrations of ethylene glycol or triethylene glycol monobutyl ether. Below the region of uncertainty printing is straighforward. 
that an effective strategy to reduce the coffee-ring effect is to induce an additional inward Marangoni flow within an evaporating droplet, which can compensate the outward Marangoni flow, enabling the formation of a uniform deposition of the ink-particles. ${ }^{4}$ This is possible by the use of a higher boiling point solvent with lower surface tension such as ethylene glycol (EG) or triethylene glycol monobutyl ether (TGME).

Figure $\mathbf{S 2}$ shows the surface tension for various mixtures of water with EG and TGME, it was found that jetting was most easily achieved for surface tensions that were below $50 \mathrm{mNm}^{-1}$ and only limited jetting was achieved above this value. As such all ink formulations used for patterning in this work have a 1:1 $\mathrm{v} / \mathrm{v}$ mix of water (18.2 $\mathrm{M} \Omega . \mathrm{cm}$ ) and ethylene glycol as the solvent. It should be noted that for the inks printed in this work the surface tension will always be lower than that of pure water-ethylene glycol mixtures due to the lowering of the surface tension by the polyelectrolyte solutes as seen in Figure S3. Furthermore, the contact angle of the 1:1 EG:water mixture on a cleaned silicon wafer was measured. An average contact angle of $(9.69 \pm 0.51)^{\circ}$ was obtained. This very small value is due to the fact that cleaned silicon has a very high surface energy due to polar silanol groups at the silica surface.

After printing of the $\mathrm{Ml}$ it was necessary to wash away the excess and leave a strongly attached monolayer at the surface. This was achieved via a careful rinse and sonication in water followed by drying under a stream of nitrogen. Although patterning through jetting, phase separation and solvent evaporation is extremely rapid (of the order of a second or less), samples were typically left overnight before rinsing to ensure strong MI attachment. In future work we will more thoroughly investigate timescales required for strong $\mathrm{MI}$ attachment. For high MI concentration the washing would result in quite noticeable spreading of $\mathrm{Ml}$ over the rest of the substrate., evident in some cases by a broadening of the printed line after polymer brush growth (Figure S4). Lower $\mathrm{Ml}$ concentrations of $2 \mathrm{mg} \cdot \mathrm{ml}^{-1}$ were sufficient to keep the spreading to a minimum and create high resolution patterns.

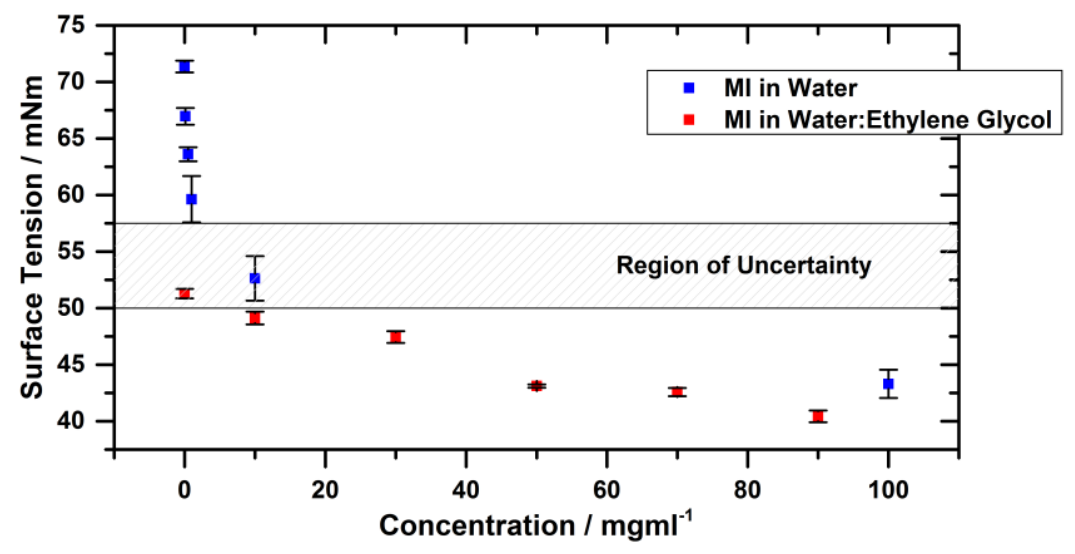

Figure S3. Surface tension of water and water:ethylene glycol (50:50 v/v\%) with varying concentration of polyeletrolyte macroinitiator. 
pHEMA

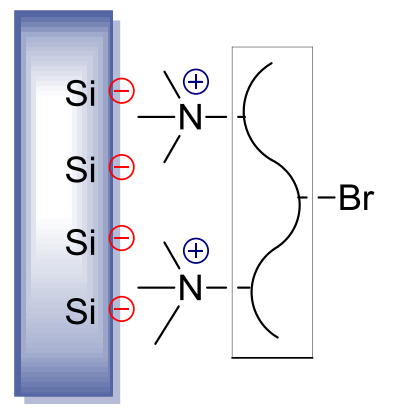

HEMA (5000 equiv.), $\mathrm{CuBr}_{2}$ (1 equiv.), bpy (10 equiv.), AA (10 equiv.) 1:1 $\mathrm{H}_{2} \mathrm{O}: \mathrm{MeOH}$, $\mathrm{RT}$, various times

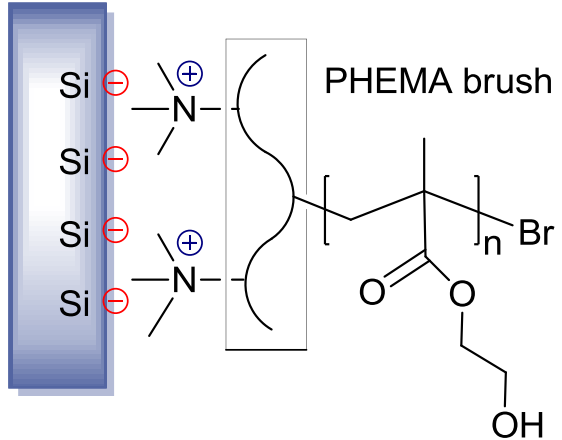

2-hydroxyethyl methacrylate $(20 \mathrm{ml}, 21.5 \mathrm{~g}, 165 \mathrm{mmol}, 5000$ equiv.), methanol $(10 \mathrm{ml})$ and Milli-Q water $(10 \mathrm{ml})$ were added to a $100 \mathrm{ml}$ round-bottomed flask and degassed by bubbling with argon for 15 minutes, before copper (II) bromide ( $7.4 \mathrm{mg}, 0.033 \mathrm{mmol}, 1$ equiv.), 2,2'-Bipyridyl (52 mg, $0.33 \mathrm{mmol}, 10$ equiv.) and ascorbic acid (58 $\mathrm{mg}, 0.33 \mathrm{mmol}, 10$ equiv.) were added, giving a dark brown solution. The mixture was degassed by bubbling for another 5 minutes and afterwards sonicated for 5 minutes to ensure all solids are dissolved. An initiator-coated silicon wafer (approximately $1 \mathrm{~cm}^{2}$ ) was washed with Milli-Q water, dried under a stream of nitrogen and placed in a glass vial $(10 \mathrm{ml})$ sealed with a septum. The vial was degassed by purging with argon for approximately 3 minutes. Approximately $4 \mathrm{ml}$ of reaction solution was syringed over the wafer and the purging was continued for another 3 minutes. The gas outlet was removed first and the argon inlet was removed at least 30 seconds later to ensure a positive pressure of argon remained. After the polymerization was allowed to proceed at room temperature for various times, the wafer was removed from the reaction solution and washed with Milli-Q water, methanol, acetone and Milli-Q water in order for at least 5 seconds each to remove any residual solution and to stop further reaction. Finally, the substrates were dried under a stream of nitrogen.
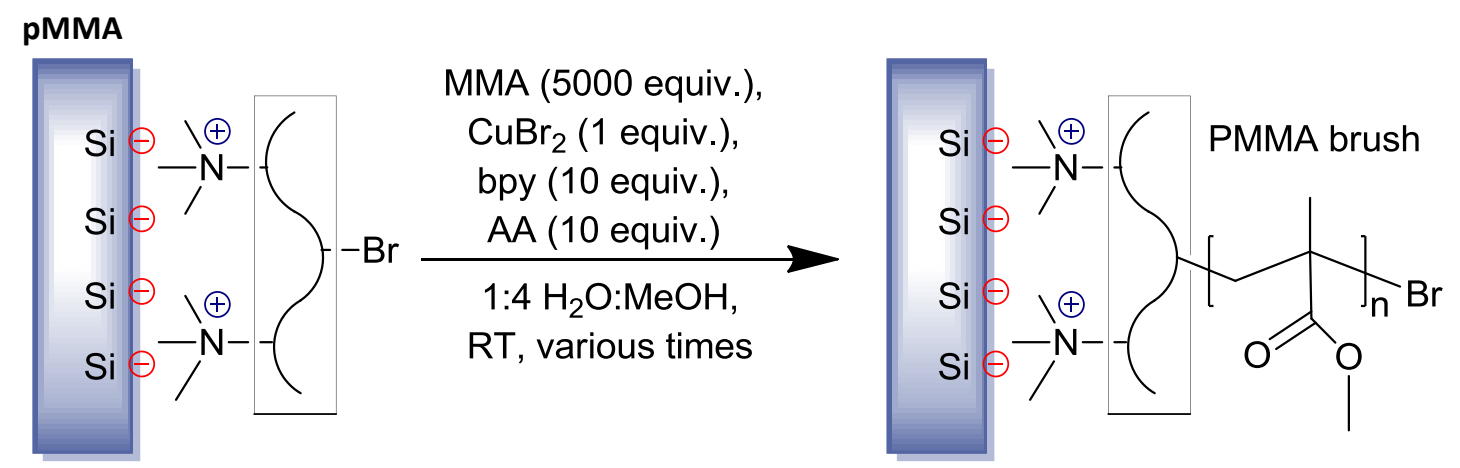

Methyl methacrylate $(20 \mathrm{ml}, 18.8 \mathrm{~g}, 187.8 \mathrm{mmol}, 5000$ equiv), methanol (16 ml) and Milli-Q water (4 ml) were added to a $100 \mathrm{ml}$ round-bottomed flask and degassed by bubbling with argon for 15 minutes, before copper (II) bromide ( $8.5 \mathrm{mg}, 0.038 \mathrm{mmol}, 1$ equiv.), 2,2'-Bipyridyl (59 mg, $0.38 \mathrm{mmol}, 10$ equiv.) and ascorbic acid ( $67 \mathrm{mg}, 0.38 \mathrm{mmol}, 10$ equiv.) were added, giving a dark brown solution. The mixture was degassed by bubbling for another 5 minutes and afterwards sonicated for 5 minutes to ensure all solids are dissolved. An Initiator-coated silicon wafer (approximately $1 \mathrm{~cm}^{2}$ ) was washed with Milli-Q water, dried under a stream of nitrogen and placed in a glass vial $(10 \mathrm{ml})$ sealed with a septum. The vial was degassed by purging with argon for approximately 3 minutes. Approximately $4 \mathrm{ml}$ of reaction solution was syringed over the wafer and the purging was continued for another 3 minutes. The gas outlet was removed first and the argon inlet was removed at least 30 seconds later to ensure a positive pressure of argon remained. After the polymerization was allowed to proceed at room temperature for various times, the wafer was removed from the reaction solution and washed with Milli-Q water, methanol, acetone and Milli-Q water in order for at least 5 seconds 
each to remove any residual solution and to stop further reaction. Finally, the substrates were dried under a stream of nitrogen.

PNIPAAM
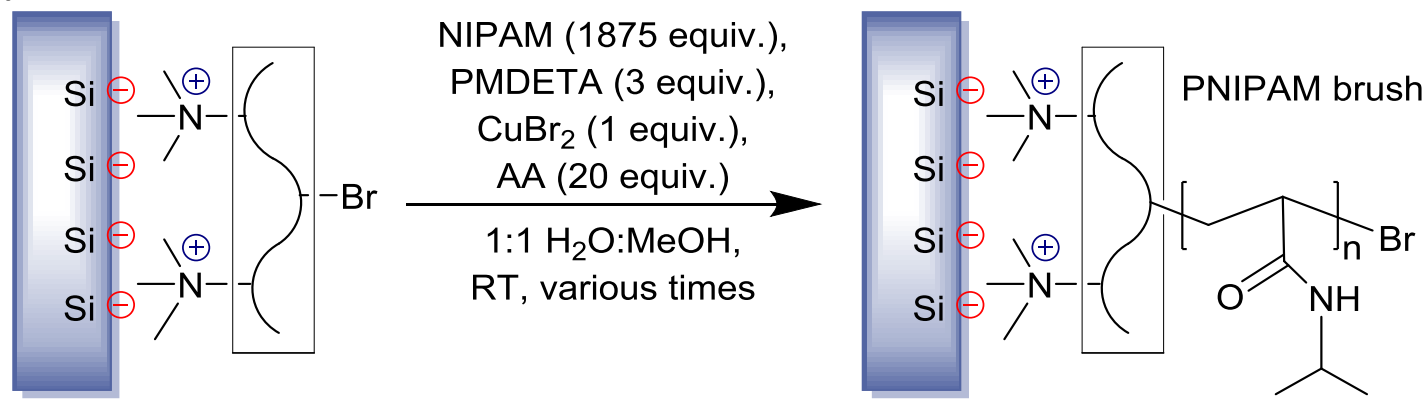

$\mathrm{N}$-Isopropylacrylamide $(6.37 \mathrm{~g}, 56.3 \mathrm{mmol}, 1875$ equiv), methanol (5 ml) and Milli-Q water (5 ml) were added to a $50 \mathrm{ml}$ round-bottomed flask and degassed by bubbling with argon for 15 minutes. Than copper (II) bromide $(6.70 \mathrm{mg}, 0.03 \mathrm{mmol}, 1$ equiv.) and Pentamethyldiethylenetriamine $(20 \mu \mathrm{l}, 16.6 \mathrm{mg}, 0.10 \mathrm{mmol}$, 3 equiv.) were added, giving a light blue/ turquoise solution. After adding ascorbic acid (106 mg, $0.60 \mathrm{mmol}$, 20 equiv.) the solution becomes colourless. The mixture was degassed by bubbling for another 5 minutes and afterwards sonicated for 5 minutes to ensure all solids are dissolved. An Initiator-coated silicon wafer (approximately $1 \mathrm{~cm}^{2}$ ) was washed with Milli-Q water, dried under a stream of nitrogen and placed in a glass vial $(10 \mathrm{ml})$ sealed with a septum. The vial was degassed by purging with argon for approximately 3 minutes. Approximately $4 \mathrm{ml}$ of reaction solution was syringed over the wafer and the purging was continued for another 3 minutes. The gas outlet was removed first and the argon inlet was removed at least 30 seconds later to ensure a positive pressure of argon remained. After the polymerization was allowed to proceed at room temperature for various times, the wafer was removed from the reaction solution and washed with Milli-Q water, methanol, acetone and Milli-Q water in order for at least 5 seconds each to remove any residual solution and to stop further reaction. Finally, the substrates were dried under a stream of nitrogen. 


\section{Supporting Data}

\section{Printing of Pure Macroinitiator Inks}

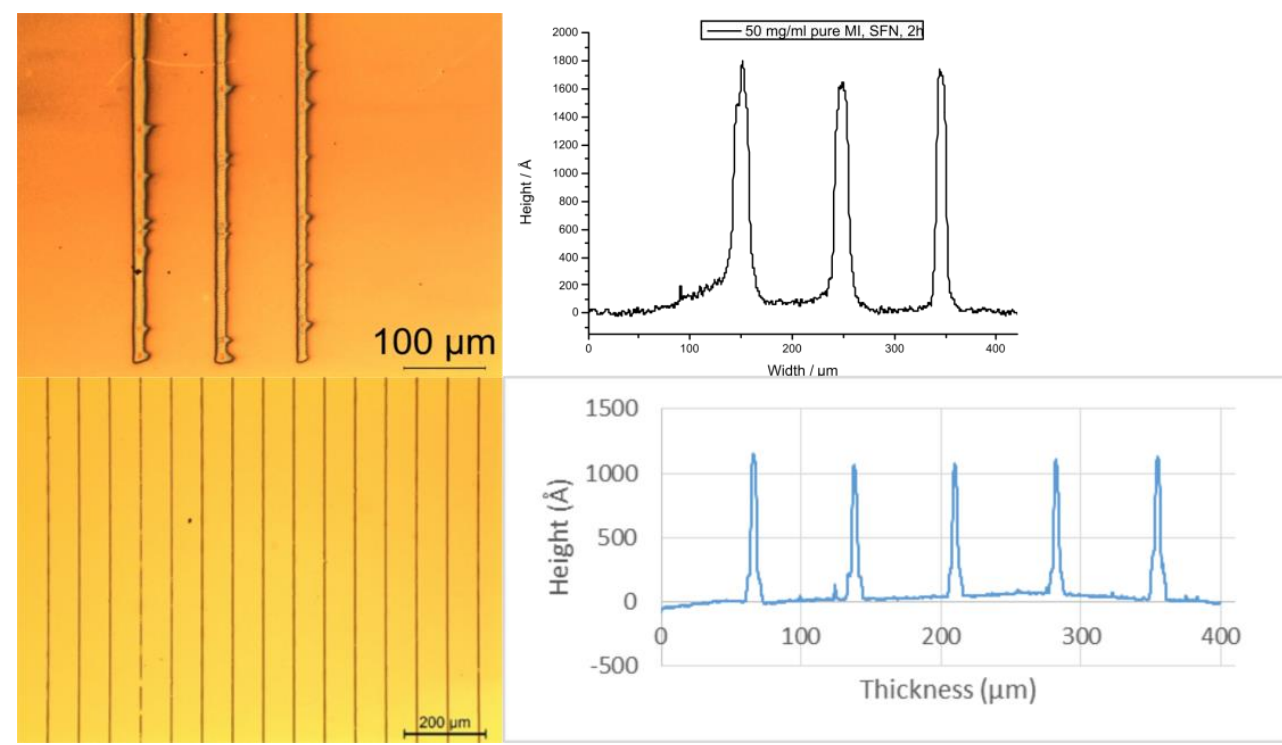

Figure S4. Optical images (left) and profilometry measurements (right) showing the effect of ink concentration on spreading of macroinitiator, top panel $\mathrm{Ml}$ concentration of 50 $\mathrm{mg} \cdot \mathrm{ml}^{-1}$ shows broadening of the lines and clear spreading of the initiator in the direction of the washing (left to right). Bottom panels show pHEMA brushes grown from a $2 \mathrm{mg} \cdot \mathrm{ml}$ ${ }^{1} \mathrm{Ml}$ concentration ink with negligible line spreading.
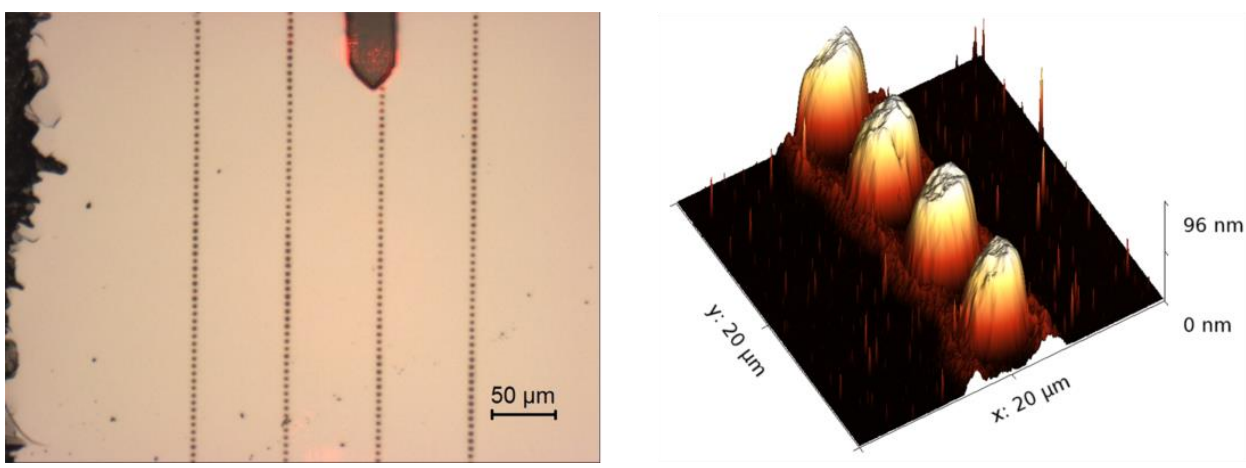

Figure S5. Single drops created using the SIJ of a $2 \mathrm{mg} \cdot \mathrm{ml}^{-1} \mathrm{MI}$ ink followed by pHEMA brush growth. Optical (left) and AFM (right). 


\section{Humidity Sensor based on Parallel Printed MI Lines}

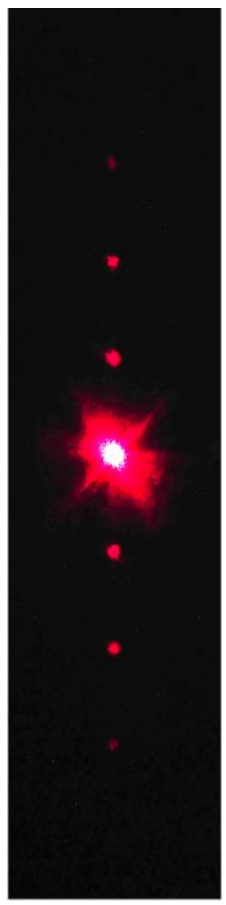

Dry

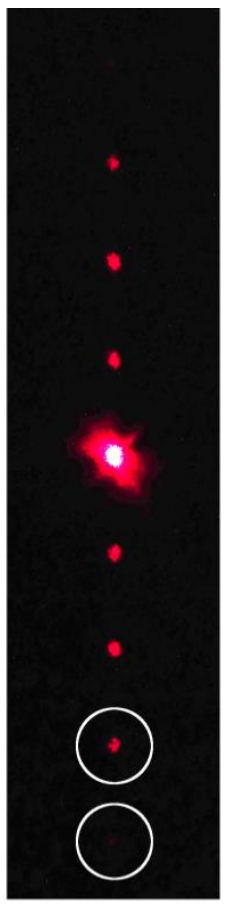

Humid

Figure S6. Diffraction pattern observed by reflection of a $650 \mathrm{~nm}$ laser from SIJ-patterned pHEMA brush lines of approximately $50 \mu \mathrm{m}$ pitch. The well-defined diffraction pattern confirms the high quality and repeatability of the patterning. The 'dry' measurement was conducted under ambient laboratory conditions, while the 'humid' measurement was conducted by condensing moisture from warm, humid air onto the sample. It is clear in the 'humid' measurement that the swelling of the polymer lines has caused intensity to be transferred from the central reflected spot to the diffracted spots (see particularly the circled spots). By measuring the intensity of a diffracted spot, this patterned sample can act as a simple humidity sensor.

Spot separaration was $30 \pm 1 \mathrm{~mm}$ at a distance of $222 \pm 1 \mathrm{~cm}$ from the sample, consistent with the targeted line pitch through the grating equation $(d \sin \theta=n \lambda)$. Images are photographs of the screen taken with a digitial camera using identical settings (aperture, shutter speed and sensitivity), subjected to identical linear adjustments of brightness and contrast, and identical digital rotation for clarity. 


\section{Supporting Data for Sub-Micron Patterning}
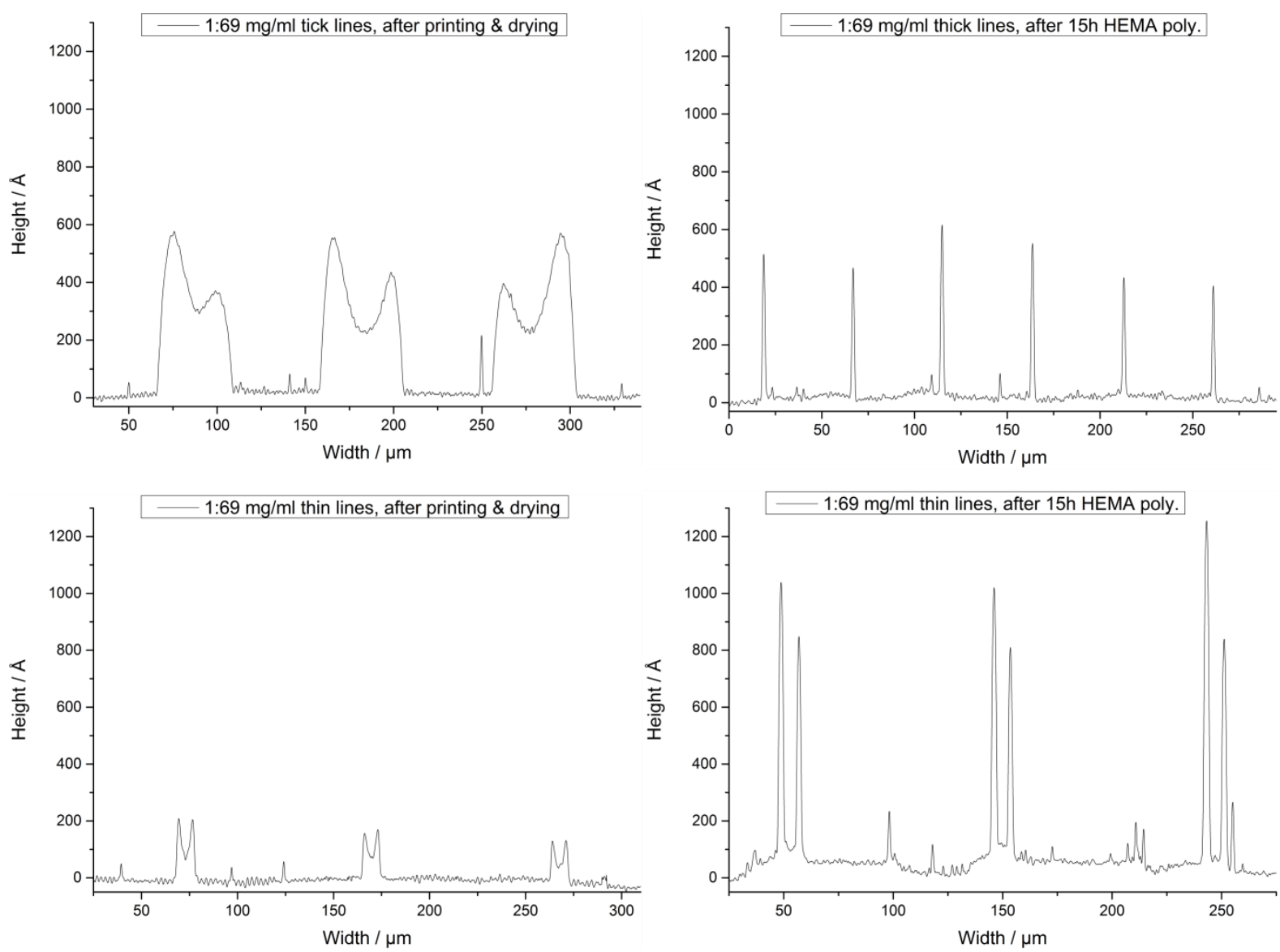

Figure S7. Profilometry measurements of printed MI:MADQUAT lines after printing (before washing) showing some signs of coffee-staining, but not significant enough to result in complete separation to the contact edge for pHEMA brush growth. Right panels show pHEMA at contact edge only after washing and polymerisation. Cases shown for wide and narrow line widths (top and bottom respectively).

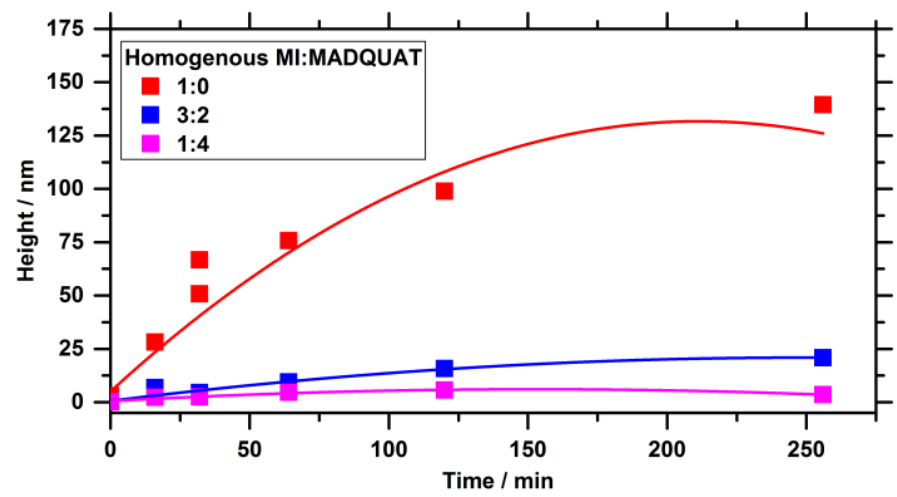

Figure S8. Effect of mixed MI:MADQUAT on the ellipsometric thickness of pHEMA brushes grown on unpatterned solution deposited macroinitiators. It is clear that diluting the MI with MADQUAT greatly reduces the grafting density and so brush thickness. 


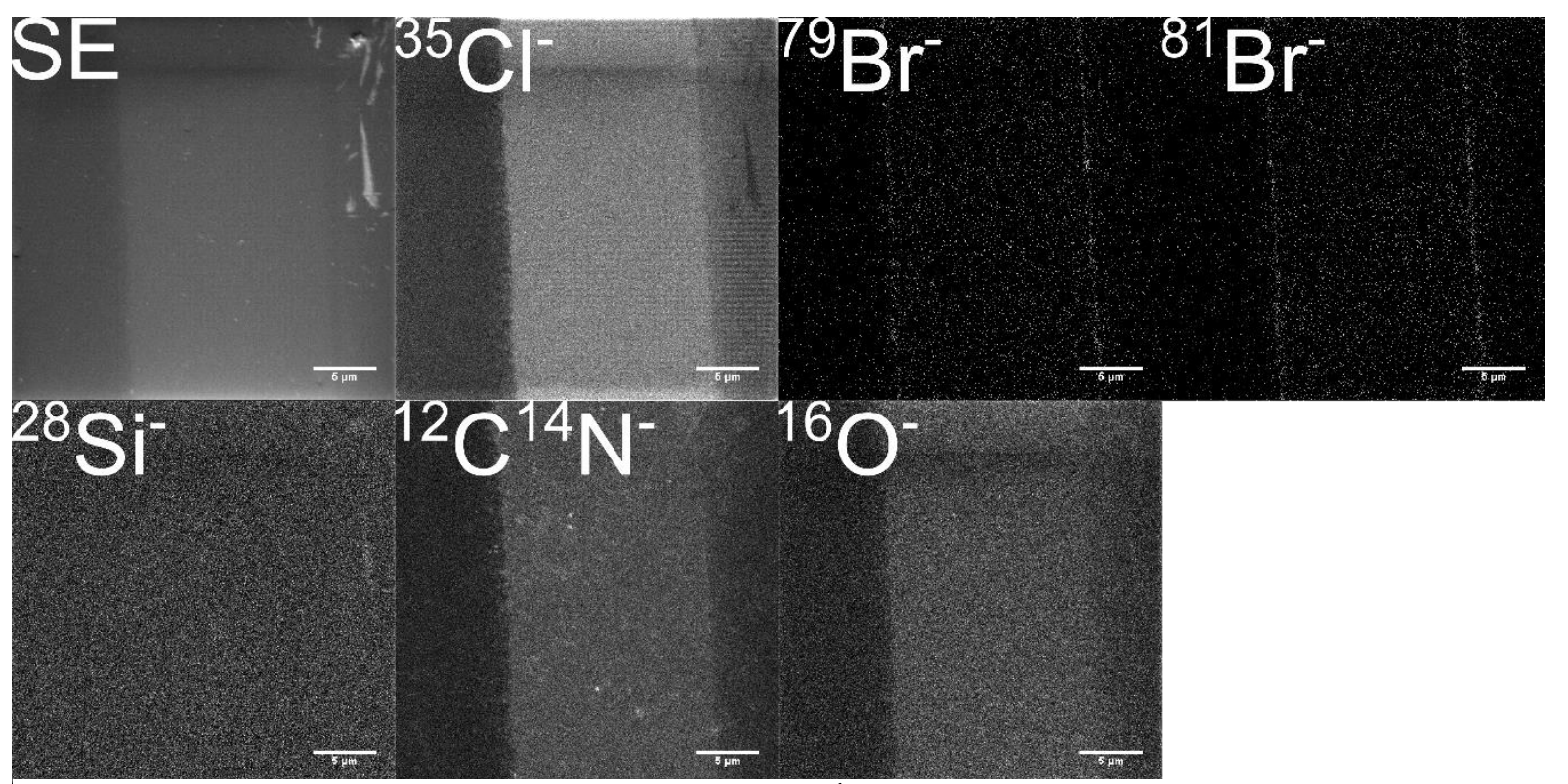

Figure S9. NanoSIMS imaging of MI:MADQUAT $\left(1: 69 \mathrm{mg} \cdot \mathrm{ml}^{-1}\right)$ printed monolayers on silicon substrates. SE = secondary electron.
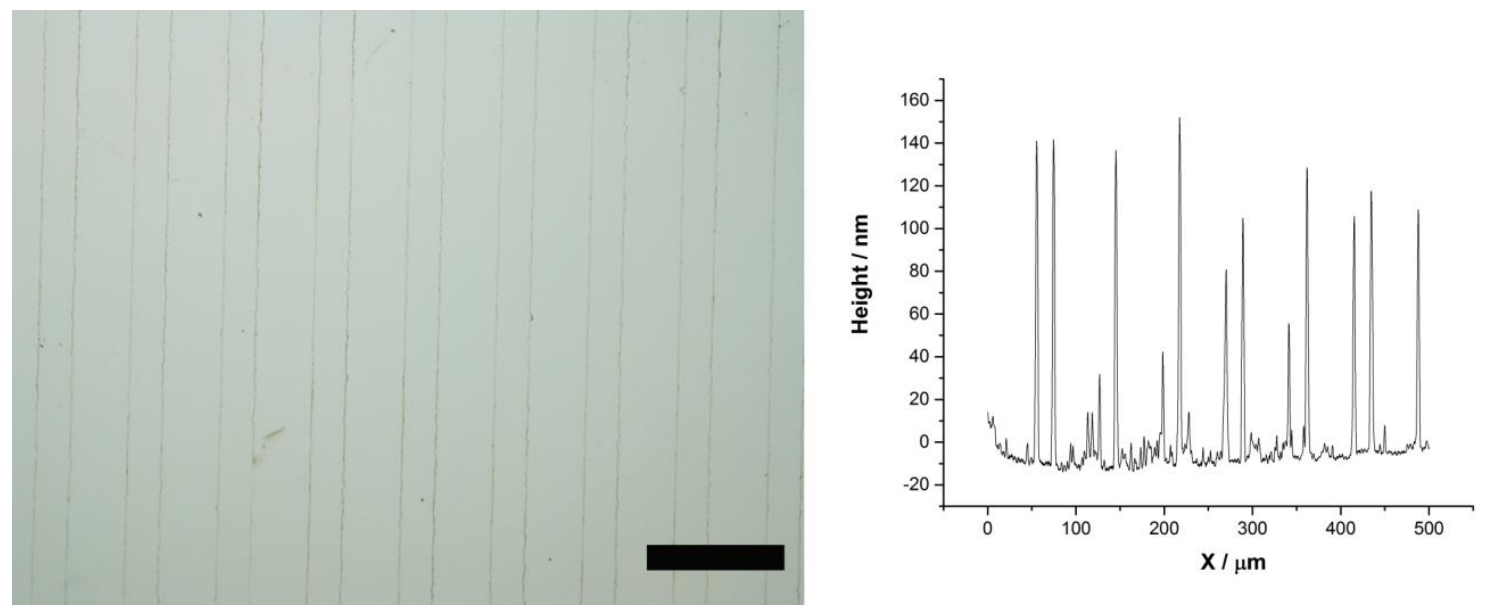

Figure S10. pHEMA brushes grown from 1:69 $\mathrm{mg} \cdot \mathrm{ml}^{-1} \mathrm{MI}$ :MADQUAT inks in pure ethylene glycol showing the phase separation for this single solvent system. 


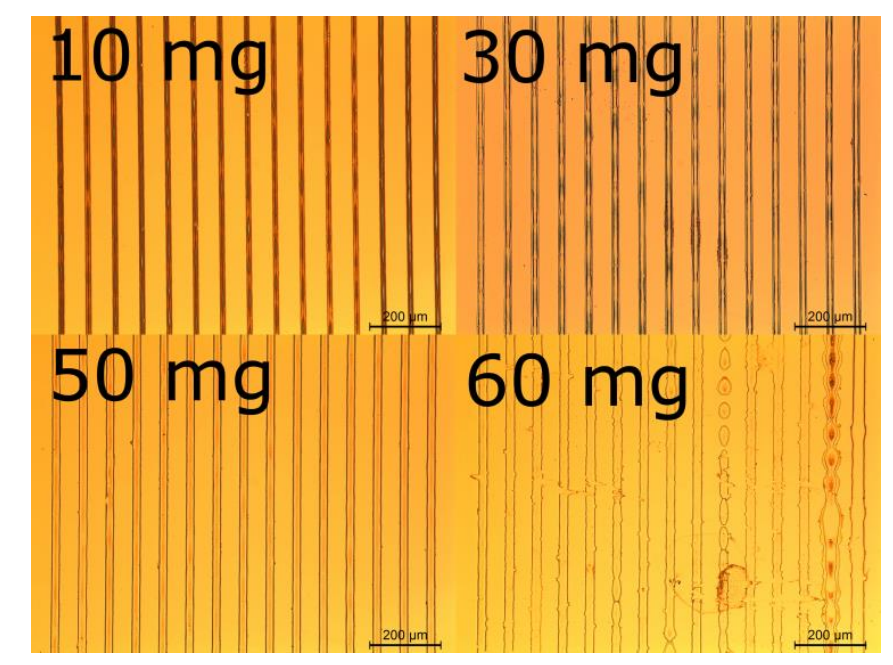

Figure S11. Microscopy images of pHEMA brushes grown from $\mathrm{MI}: \mathrm{NaCl}$ inks printed with SIJ showing the evolution of phase separation to the contact edge with increasing salt concentration $(0.17 \mathrm{M}$, $0.51 \mathrm{M}, 0.86 \mathrm{M}$ and $1.03 \mathrm{M}$ respectively) and a constant $\mathrm{Ml}$ concentration of $2 \mathrm{mg} \cdot \mathrm{ml}^{-1}$.

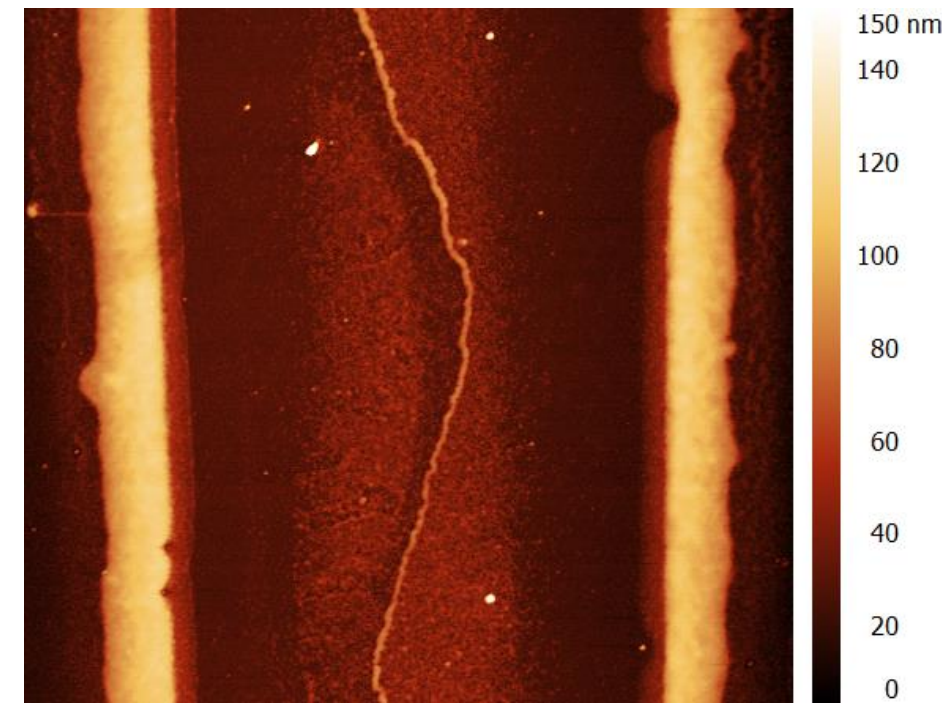

Figure S12. Atomic force microscopy image of pHEMA brushes grown from a $2 \mathrm{mg} \cdot \mathrm{ml}^{-1} \mathrm{MI}$ and $1.03 \mathrm{M}$ $\mathrm{NaCl}$ in solution. The printed line shows the segregation effect in $\mathrm{MI}$ simple salt solutions. 


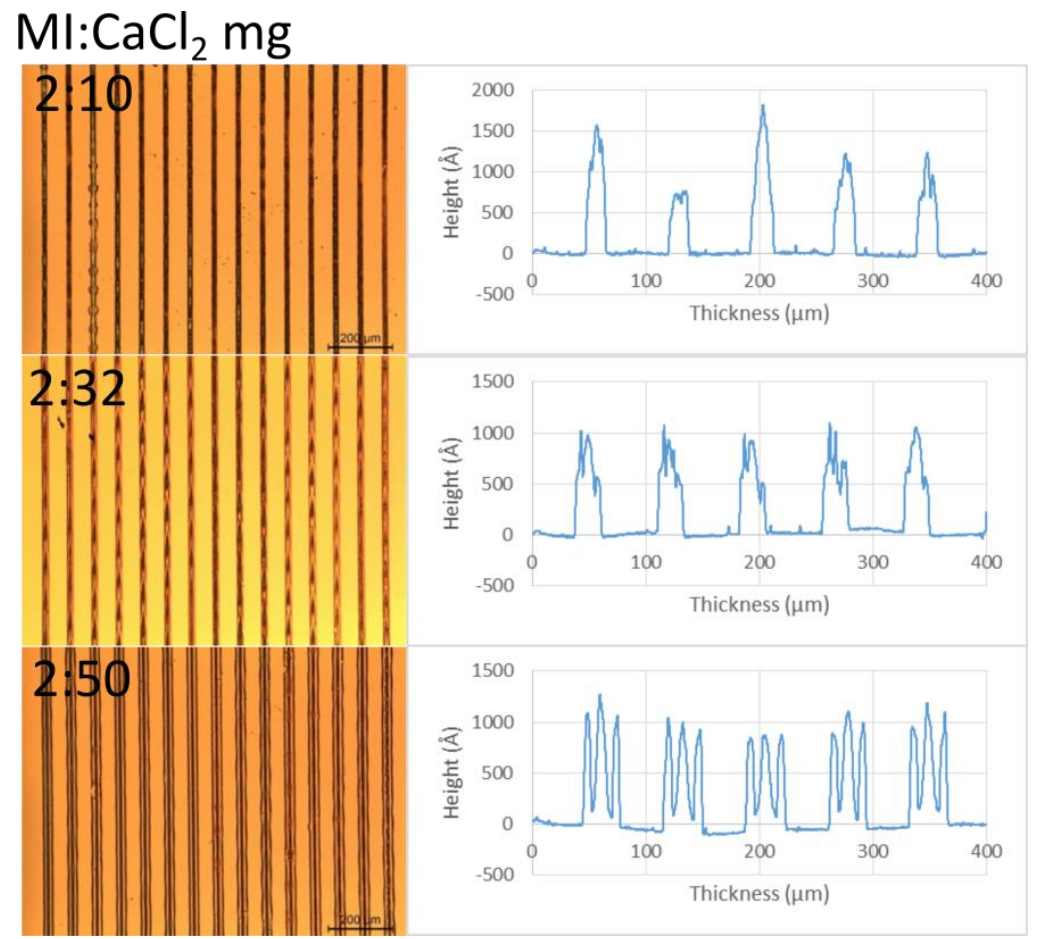

Figure S13. Microscopy and stylus profiles for mixed $\mathrm{MI}: \mathrm{CaCl}_{2}$ inks printed with SIJ. Influence on $\mathrm{MI}$ phase separation is less for $\mathrm{CaCl}_{2}$ (concentration $0.09 \mathrm{M}, 0.29 \mathrm{M}$ and $0.45 \mathrm{M}$ from top to bottom) than for MADQUAT but similar to $\mathrm{NaCl}$ (Figure S11, Figure 4). $\mathrm{Ml}$ at $2 \mathrm{mg} \cdot \mathrm{ml}^{-1}$. 
MI:pDADMAC / $\mathrm{mgml}^{-1}$
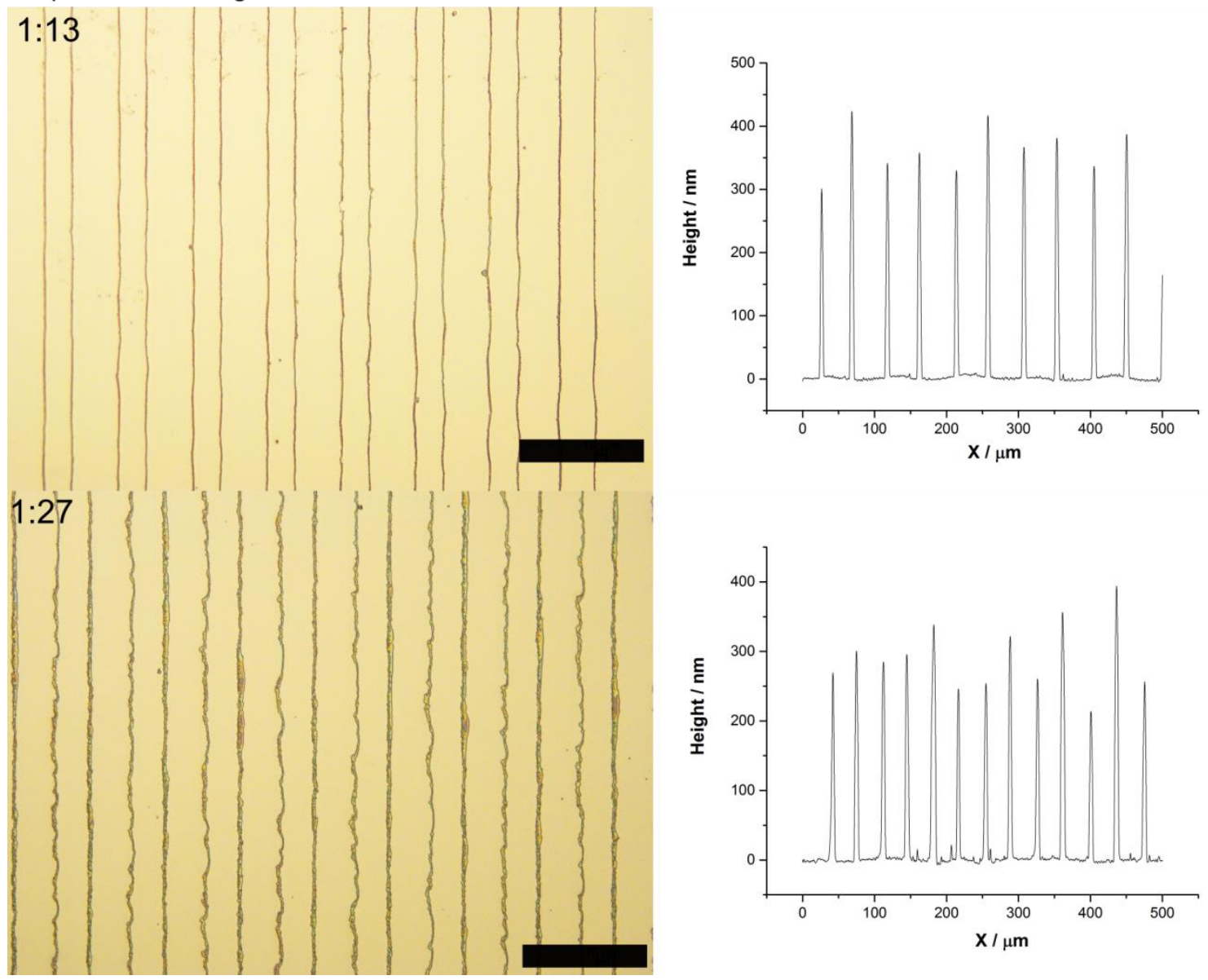

Figure S14. pHEMA brushes grown from macroinitiators printed from MI:pDADMAC inks in ethylene glycol / water showing the same phase separation and sub-micron patterning from MI:MADQUAT inks. Scale bar $100 \mu \mathrm{m}$. It is likely that the lines are less well-defined compared to those produced with MADQUAT due to printing conditions (e.g. solvent, surface tension, voltage, nozzle size) not being optimised for this polymer. 


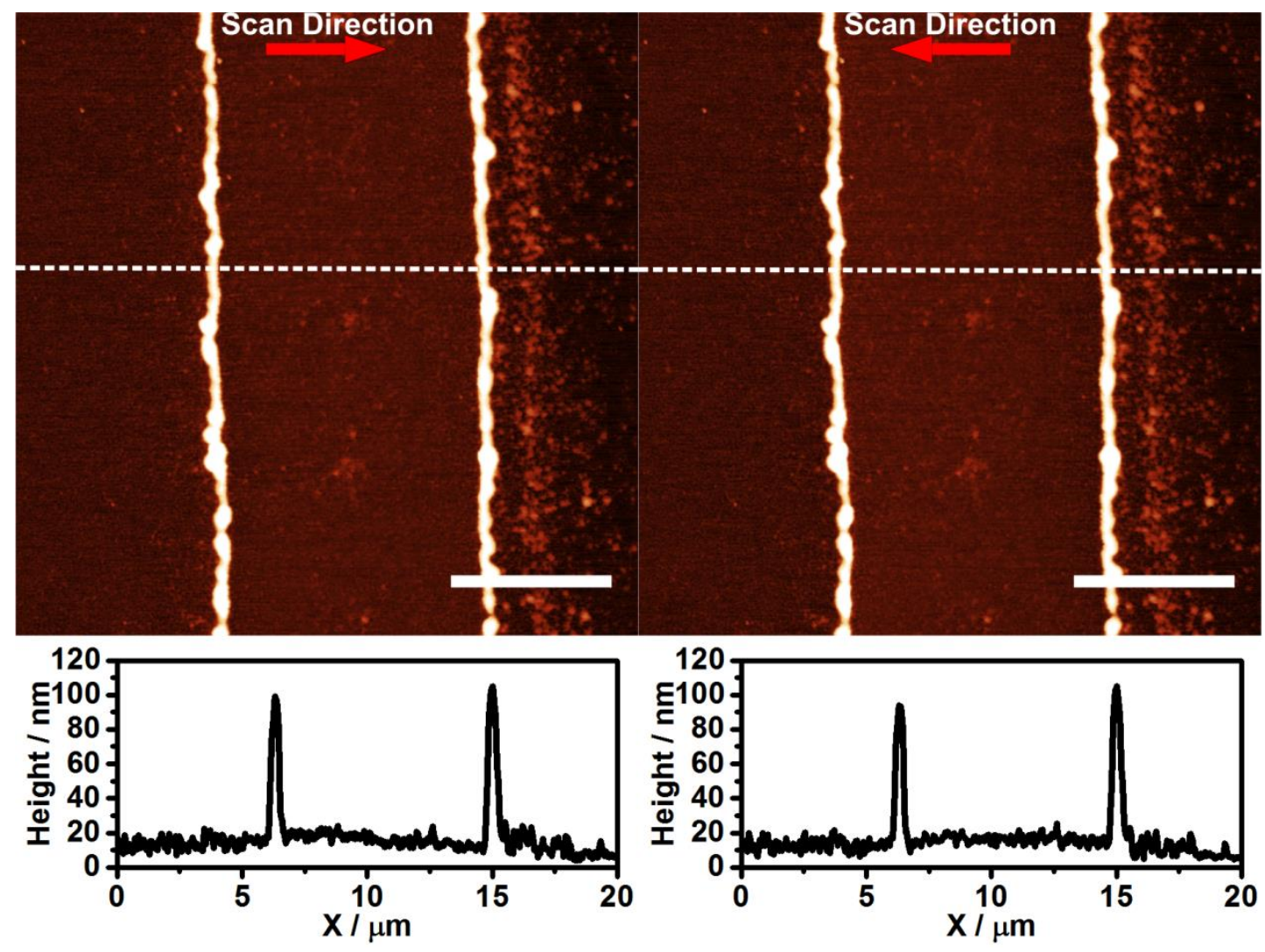

Figure S15. Atomic force microscopy trace and retrace images of submicron features demonstrating accurate reproduction of surface topology independant of scan direction. Scale bars are $5 \mu \mathrm{m}$. The effect of tip aspect ratio on imaging is discussed in the Materials and Methods section above. This image is a larger version of the 1:69 MI:MADQUAT sample image shown in Fig 3 in the main text. 


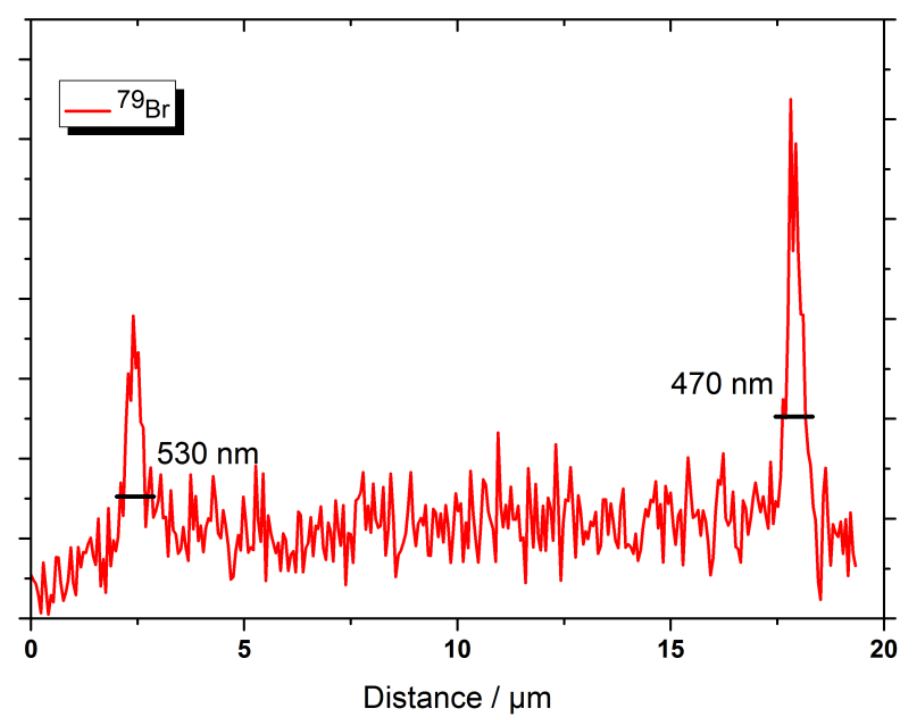

Figure S16. NanoSIMS line profile (identical data to Fig 2c in the main text) confirming the sub-micron feature widths measured by AFM.

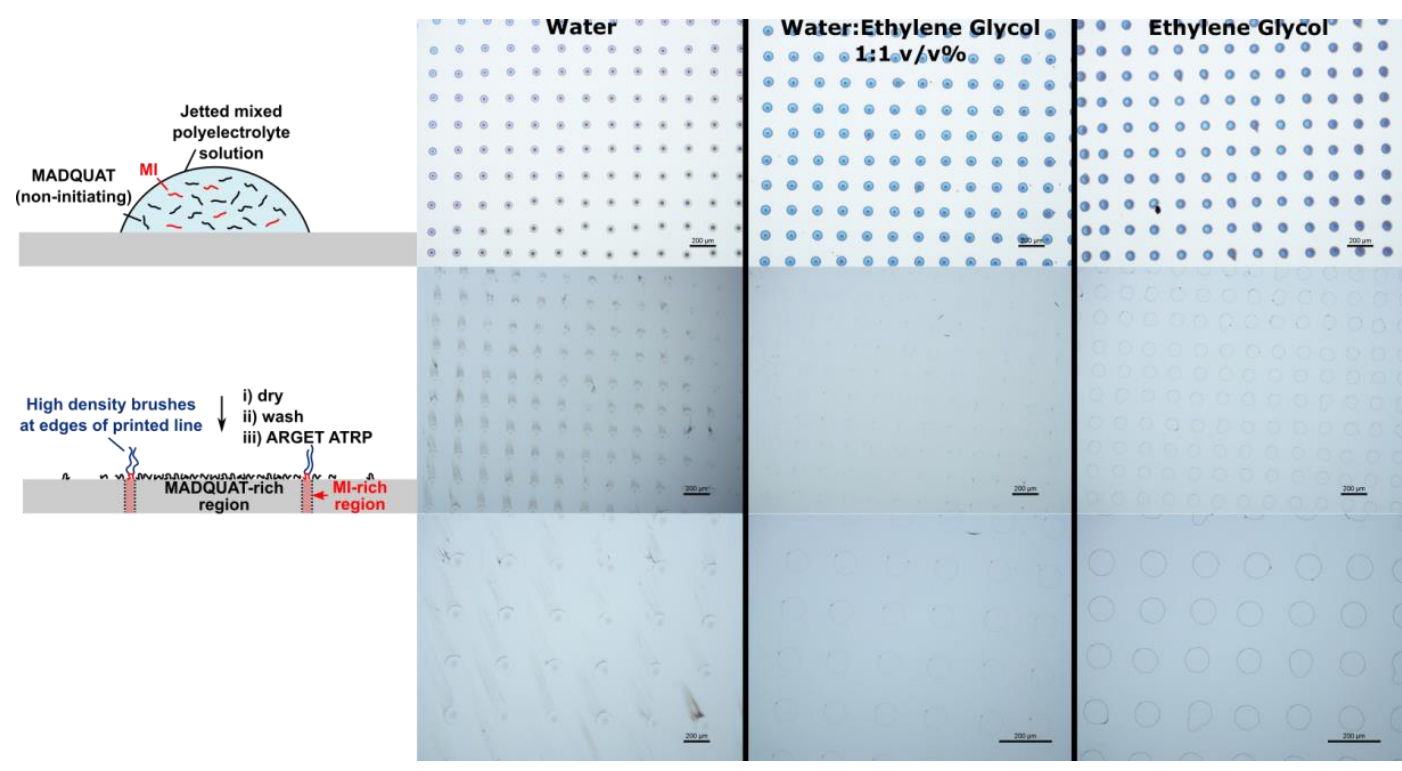

Figure S17. Top: 1:69 $\mathrm{mgml}^{-1} \mathrm{MI}$ :MADQUAT inks printed with a piezoelectric printer (DimatixDMP2831). Middle and bottom: polymer brush features formed after rinsing and 23 hours pHEMA polymerisation. Inks with varying dielectric constants have been used: Water $(\varepsilon=79.6), 1: 1$ water:ethylene glycol ( $\varepsilon=64.8)$ and ethylene glycol $(\varepsilon=39)$. All samples show patterning due to phase separation, although using water as a solvent leads to additional artefacts (a central feature in each ring and significant spreading on rinsing). This suggests our patterning effect is not dependent on exact solvent polarity. Furthermore, using a piezoelectric printer rules out the electrostatic mechanism of SIJ printing as the origin of the patterning. 
Table S1. This table demonstrates the method for calculating the ionic strength (I) of the solutions and the corresponding Debye screening lengths $\left(\kappa^{-1}\right)$. Ionic strength was calculated using the formula:

$I=\frac{1}{2} \sum_{i=1}^{i} c_{i} z_{i}^{2}$

where $c_{i}$ is the molar concentration of ion $i$ and $z_{i}$ is the charge number on $i$. The Debye length was calculated using the equation

$\kappa^{-1}=\sqrt{\frac{\varepsilon_{r} \varepsilon_{0} k_{B} T}{2 N_{A} e^{2} I}}$

where $\varepsilon_{0}$ is the permitivity of free space, $k_{B}$ is the Boltzmann constant, $T$ is the temperature in kelvin, $N_{A}$ is Avagadros number and $\mathrm{I}$ is the ionic strength in $\mathrm{mol} \cdot \mathrm{m}^{-3} \cdot \varepsilon_{\mathrm{r}}$ is the dielectric constant of the surrounding media, which, for aqueous ethylene glycol solutions at $20^{\circ} \mathrm{C}$ can be calculated from the following equation (5):

$\varepsilon_{r}=79.752-0.18962 x-0.0021824 x^{2}$

where $\boldsymbol{x}$ is the weight percent of ethylene glycol in the solution. Corresponding to dielectric constants of

$\varepsilon_{\mathrm{rH}_{2} \mathrm{O}}=79.6$

$\varepsilon \mathrm{rH}_{2} \mathrm{O}: \mathrm{EG}=64.8$

$\varepsilon_{\mathrm{rEG}}=39.0$

The molecular weight for MADQUAT and pDADMAC and is taken as the repeat unit in the ionic strength calculations. The polymer molecular weight for MADQUAT is unknown (Sigma 657670), the polymer molecular

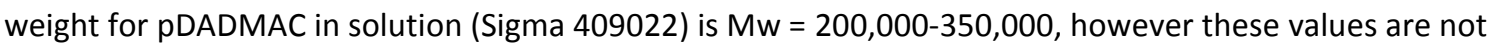
necessary for the calculation.

The molecular weight for the $\mathrm{Ml}$ is the molecular weight of the statistical copolymer containing 65 ionic groups and 29 initiator groups using the structure shown in Fig S1. Synthetic details for this polymer are given above.

This table shows ionic strengths for mass concentration $\rho_{i}=1 \mathrm{mg} \cdot \mathrm{ml}^{-1}$ solutions, and corresponding Debye screening lengths.

\begin{tabular}{|c|c|c|c|c|c|c|c|c|c|c|c|}
\hline & $\begin{array}{l}\mathrm{M} \\
\mathrm{g} \cdot \mathrm{mol}^{-1}\end{array}$ & $\begin{array}{l}\rho_{\mathrm{i}} \\
\mathrm{mg} \cdot \mathrm{ml}^{-1}\end{array}$ & $\begin{array}{l}\mathrm{c}_{\mathrm{i}} \\
\mathrm{mM}\end{array}$ & $z_{1}$ & $\begin{array}{l}\text { Cha } \\
\mathrm{z}_{2} \\
\end{array}$ & $\begin{array}{c}\text { ges } \\
\#_{1} \\
\end{array}$ & $\#_{2}$ & $\begin{array}{l}\mathrm{I} \\
\mathrm{mol} \cdot \mathrm{m}^{-3}\end{array}$ & $\begin{array}{l}\kappa^{-1} \mathrm{H}_{2 \mathrm{O}} \\
\mathrm{nm}\end{array}$ & $\begin{array}{l}\mathbf{K}^{-1}{ }_{\text {H2O:EG }} \\
\mathrm{nm}\end{array}$ & $\begin{array}{l}\kappa^{-1} \\
\mathrm{~nm}\end{array}$ \\
\hline $\mathrm{NaCl}$ & 58.44 & 1 & 17.11 & 1 & -1 & 1 & 1 & 17.11 & 2.33 & 2.10 & 1.63 \\
\hline $\mathrm{CaCl}_{2}$ & 110.98 & 1 & 9.01 & 1 & -1 & 1 & 2 & 13.52 & 2.62 & 2.36 & 1.83 \\
\hline MADQUAT & 207.5 & 1 & 4.82 & 1 & -1 & 1 & 1 & 4.82 & 4.38 & 3.95 & 3.06 \\
\hline pDADMAC & 161.5 & 1 & 6.19 & 1 & -1 & 1 & 1 & 6.19 & 3.87 & 3.49 & 2.70 \\
\hline MI & 26769.5 & 1 & 0.04 & 1 & -1 & 65 & 65 & 2.43 & 6.18 & 5.57 & 4.32 \\
\hline
\end{tabular}




\section{Polymer Brush Patterning}

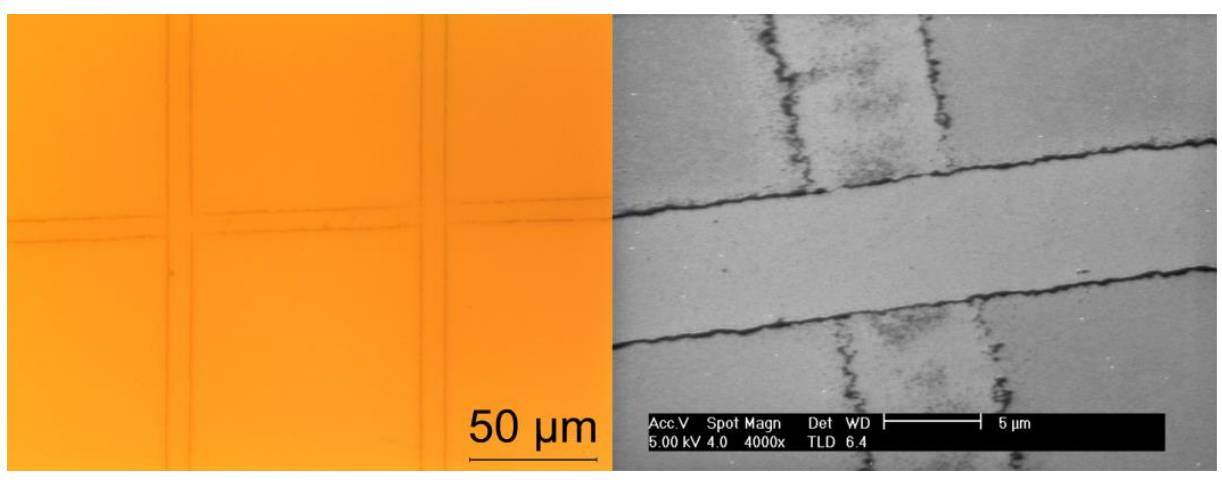

Figure S18. Optical and scanning electron micrograph of polymer brush cross. The first line (vertical in OM and horizontal in SEM) was printed several days before the second to allow sufficient attachment of the macroinitiator to the surface. This is evidence for the presence of polyelectrolyte (MADQUAT) at the center of the line attached to the silicon, preventing the deposition of any new material.

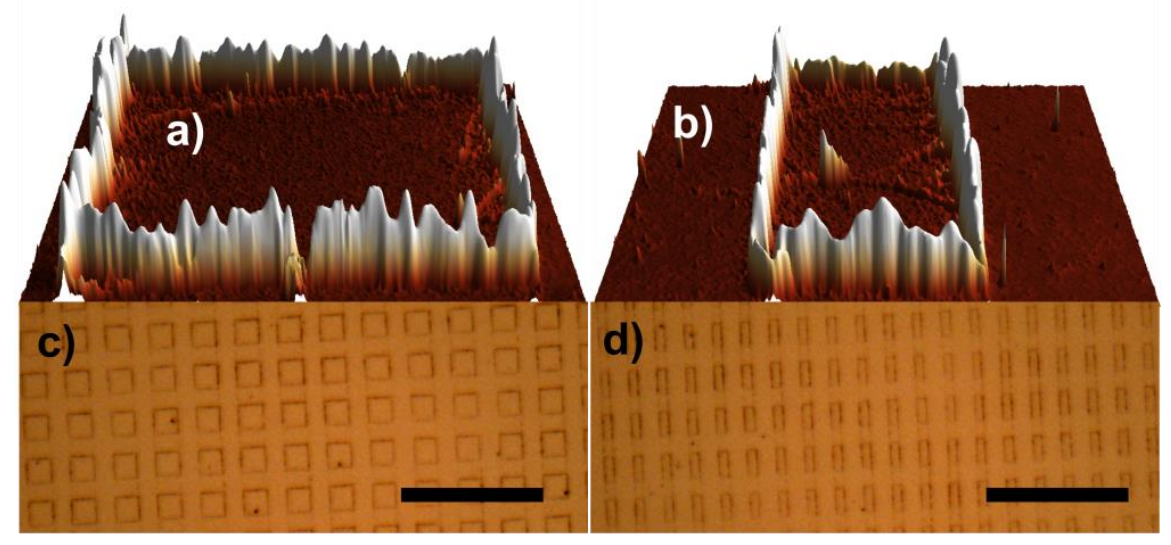

Figure S19. Boxes are created by the printing of a crosshatch pattern without allowing time for the macroinitiator to bind to the surface. Polyelectrolyte is re-dissolved during the subsequent perpendicular passes and re-deposited to the edge of the newly printed line. This results in boxes where the center is the unmodified silicon (apart from adventitious MI spread from the walls during washing), the walls are polymer brush and between them is the polyelectrolyte MADQUAT. Scale bar $50 \mu \mathrm{m}$. 

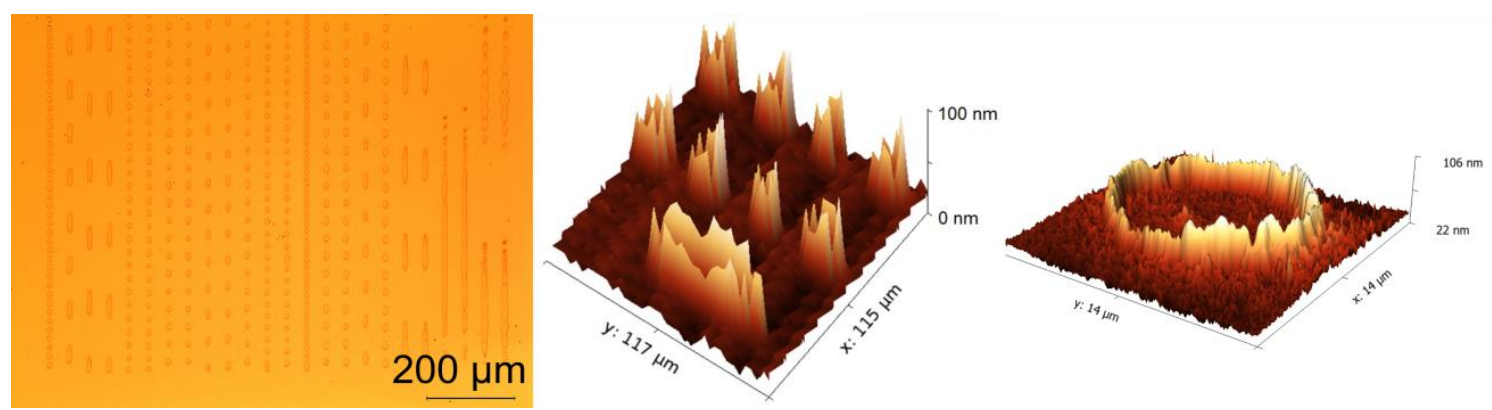

Figure S20. Cylinders of various shapes and sizes created by varying SIJ printing parameters. i.e. speed, frequency, voltage and using a bump mode to create individual droplets.

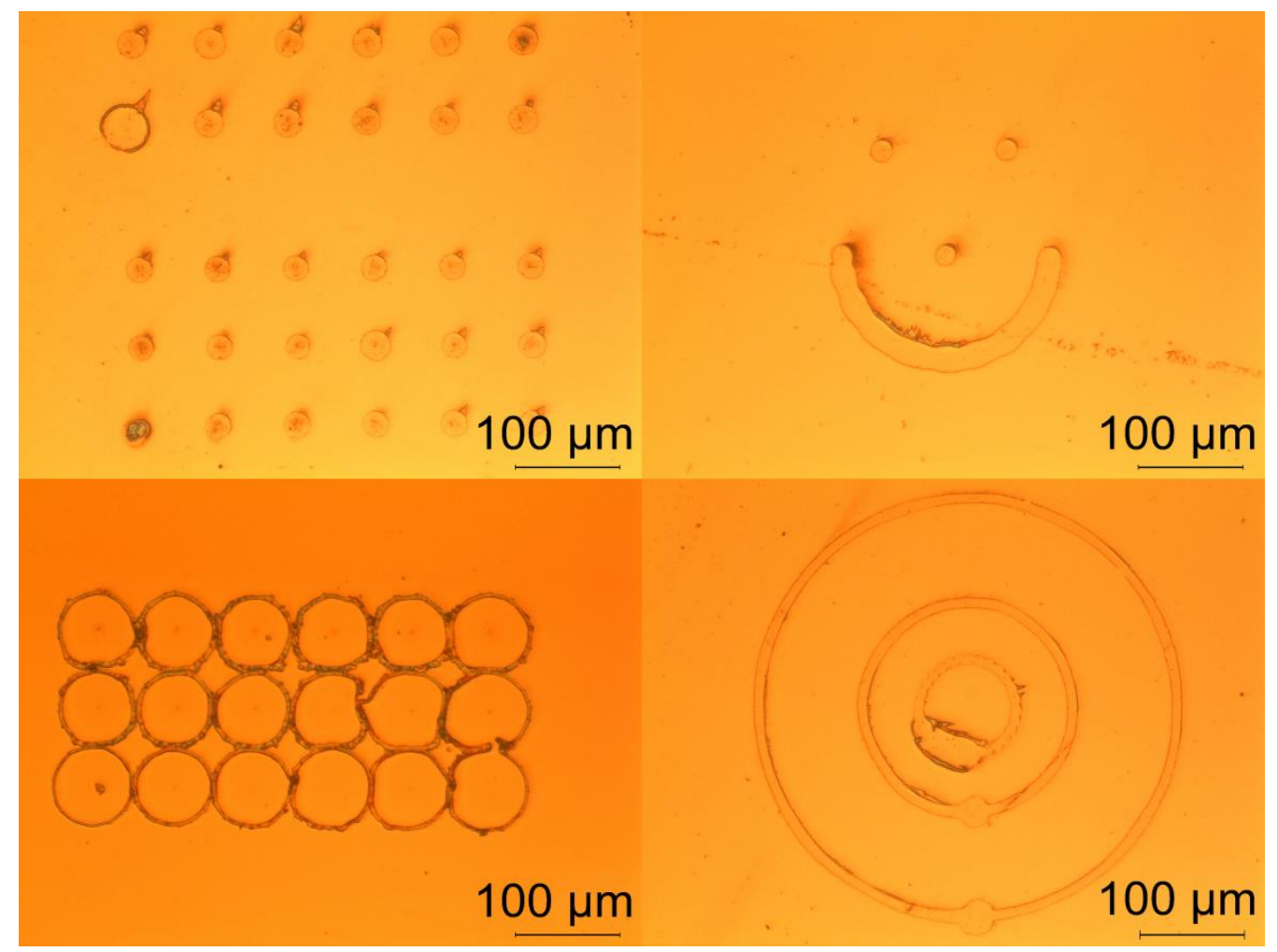

Figure S21. Other patterns. Drops inside drops (top left), smiley (top right), rings (bottom left) and concentric rings (bottom right). 


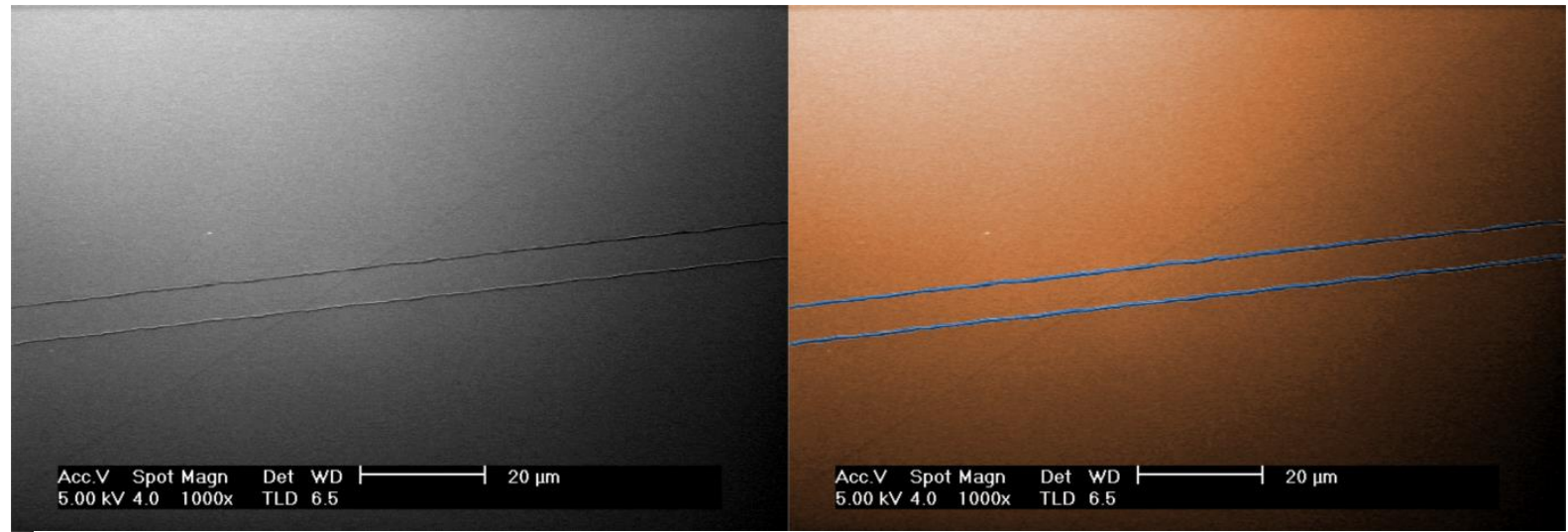

Figure S22. SEM phase separated pHEMA sub-micron lines. Right image was colourized using GIMP, image used for ToC Graphic. 


\section{Synthesis of Different Polymers and Block-co-Polymers}
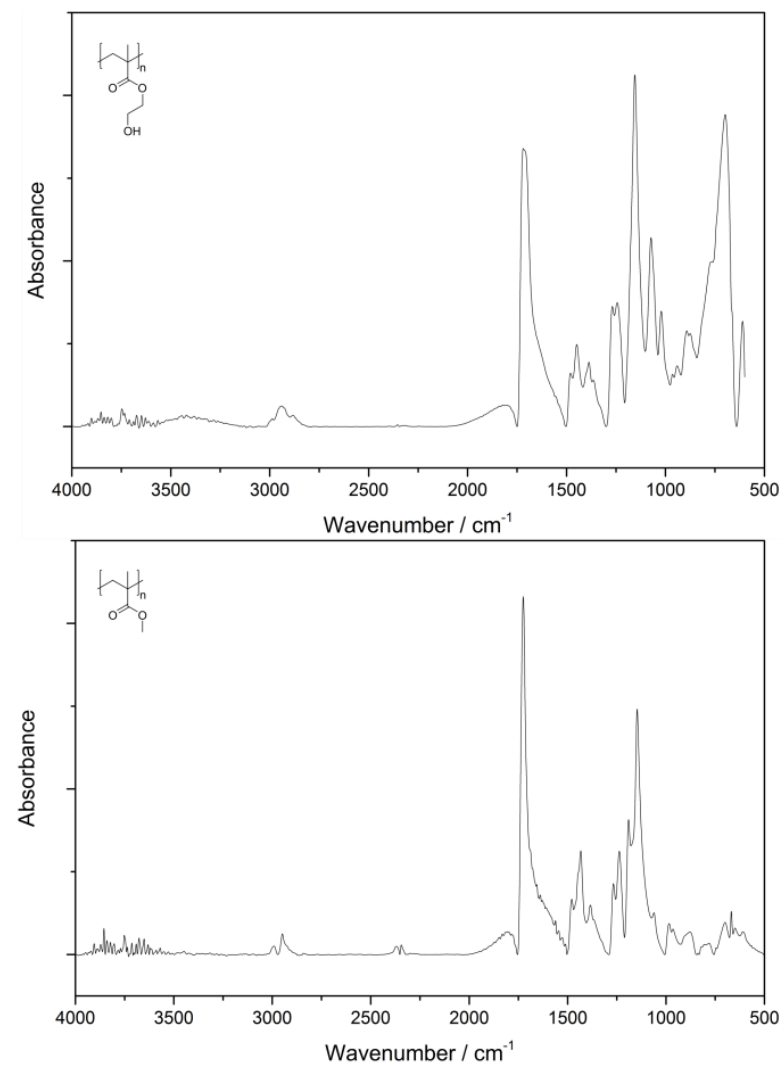

Figure S23. ATR-FTIR of pHEMA and pMMA grown by ARGET-ATRP.
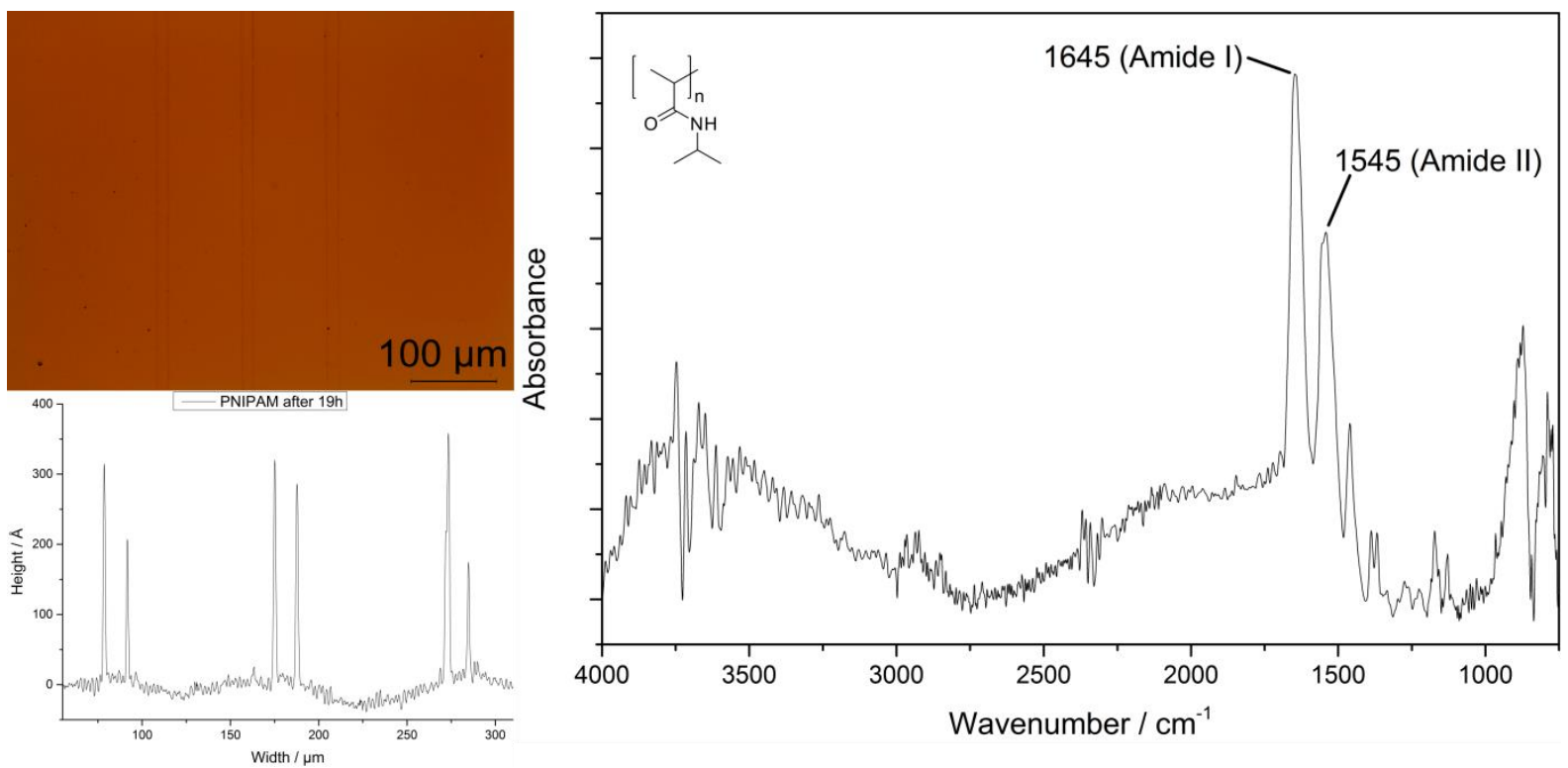

Figure S24. Growth and FTIR of pNIPAAM into phase separated sub-micron patterns. Optical microscopy, stylus profilometry and ATR-FTIR. 

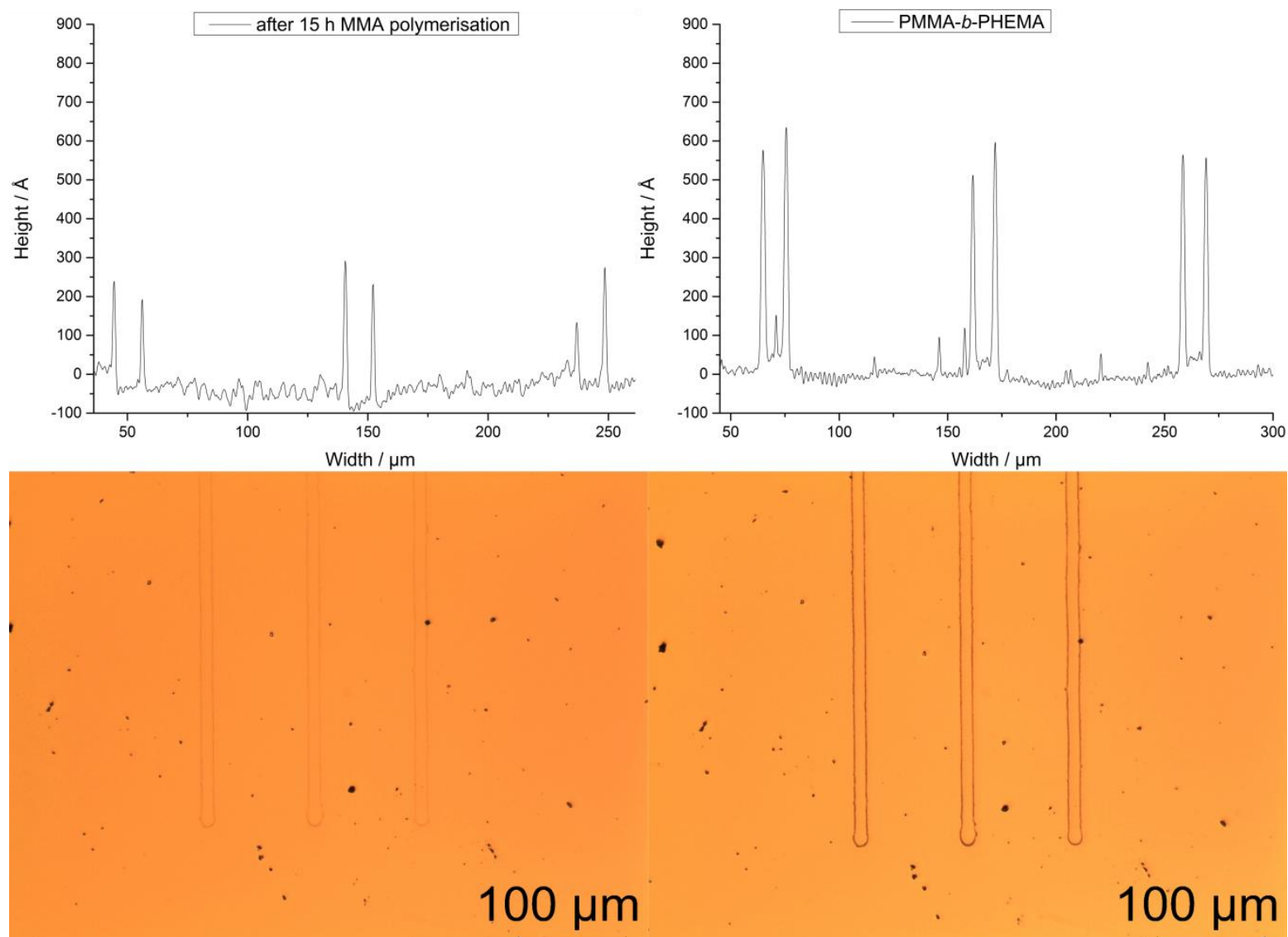

Figure S25. Growth of a block copolymer brush of pMMA-b-pHEMA, demonstrated by increase in pattern height after the second block is grown. 


\section{Supporting Information References}

(1) Cheesman, B. T.; Willott, J. D.; Webber, G. B.; Edmondson, S.; Wanless, E. J. ACS Macro Lett. 2012, 1, 1161.

(2) Fielding, L. a.; Edmondson, S.; Armes, S. P. J. Mater. Chem. 2011, 21, 11773.

(3) Chen, X. Y.; Armes, S. P.; Greaves, S. J.; Watts, J. F. Langmuir 2004, 20, 587.

(4) Kuang, M.; Wang, L.; Song, Y. Adv. Mater. 2014, 26, 6950.

(5) ME Global Ethylene Glycol Product Guide.

http://www.meglobal.biz/media/product_guides/MEGlobal_MEG.pdf (accessed Jul 7, 2016) 